\title{
NORMAL CURVES ARISING FROM LIGHT OPEN MAPPINGS OF THE ANNULUS( $\left.{ }^{(}\right)$
}

\author{
BY \\ MORRIS L. MARX
}

1. Introduction. A mapping $\delta$ of an oriented one-dimensional manifold $J$ into the complex plane $E^{2}$ is called a representation; if $\delta$ possesses a continuous nonvanishing tangent $\delta^{\prime}$, then $\delta$ is called a regular representation. An image point $\delta_{0}$ of a regular curve $\delta$ is a vertex if there exist exactly two distinct points $x$ and $y$ such that $\delta(x)=\delta(y)=\delta_{0}$ and if the tangents $\delta^{\prime}(x)$ and $\delta^{\prime}(y)$ are linearly independent. A regular curve is normal if it has a finite number of vertices and every other image point has but one pre-image. Two representations (regular representations) $\delta$ and $\varepsilon$ are equivalent if there exists a sense-preserving homeomorphism $\phi: J \rightarrow J$ such that $\varepsilon=\delta \circ \phi$ (and $\phi^{\prime}$ is continuous and nonvanishing). A regular (normal) curve is then defined to be an oriented curve with a regular (normal) representation.

Suppose $D$ is an open subset of a two-dimensional manifold and $D$ is bounded by the Jordan curves $J_{1}, J_{2}, \cdots, J_{n}$. Let $\delta_{i}$ be a representation on $J_{i}$ for $i=1, \cdots, n$. A continuous function $f$ from $\bar{D}$ into $E^{2}$ is called an extension of $\delta_{1}, \cdots, \delta_{n}$ to $D$ if $f \mid J_{i}=\delta_{i}$ for $i=1, \cdots, n$. Much of the work on extensions has been done for the class of normal curves [5], [9], [10]. A possible reason for this is that well developed combinational tools are available. These tools have been used by Heins and Morse [1], Morse [2], Titus [4], [6] and Titus and Young [7] in their studies of extensions with various analytic or topological properties. In particular Titus [6] has given necessary and sufficient conditions that a normal representation have a light open extension to the disk. The methods developed by Titus are brought to bear in this paper on a related problem; an algorithm is given that yields necessary and sufficient conditions for a pair of normal curves to have a light open extension to the annulus.

2. Preliminaries. The notation used is essentially that used in [6]. For convenience a summary of the notation and results of [6] is given in this and the following section.

Presented to the Society, January 27, 1965 under the title Image of the boundary of an annulus under a light open mapping; received by the editors February 3, 1965.

(1) This research was supported in part by a National Science Foundation Summer Fellowship and National Science Foundation Grant Number GP 1634; it represents a portion of the author's dissertation submitted to the faculty of Tulane University in partial fulfillment of the requirements for the degree of Doctor of Philosophy. The author wishes to express his appreciation to Professor G. S. Young for his direction of this research. 
In what follows $\delta$ will be a representation of a closed curve. Let $[\delta]$ denote the point set consisting of image points of $\delta$. Let $w(\delta, p)$ be the index of $\delta$ about a point $p$ not in $[\delta]$.

The outer boundary of $\delta$ will be the subset of $[\delta]$ which is contained in the closure of the unbounded component of the complement of $[\delta]$; an outer point $p$ is a point on the outer boundary such that $p$ has but one pre-image. For normal $\delta$ and any nonvertex $p$ one can define $w^{+}(\delta, p)$ and $w^{-}(\delta, p)$ as the larger and smaller winding numbers of points $p^{\prime}$ near $p$ but not on $[\delta]$. An outer point $p$ is positive [negative] if $w^{+}(\delta, p)=1\left[w^{-}(\delta, p)=-1\right]$.

Let $\delta$ be normal and let $\delta(0)$ be an outer point where $\delta$ is given by the complexvalued function $\delta(t)=a(t)+i b(t)$ with $t$ the usual angle parameter, $0 \leqq t<2 \pi$. Index the $n$ vertices in the natural way by traversing the curve with increasing $t$ and using consecutively the integers $0,1, \cdots, n-1$; thus $\delta_{0}, \delta_{1}, \cdots, \delta_{n-1}[6$, Figure 1 , p. 49].

The $2 n$ pre-images of the vertices will be denoted by the lower case Roman equivalent of the Greek letter denoting the curve and they will be indexed so that $0<d_{0}<d_{1}<\cdots<d_{2 n-1}<2 \pi$. Denote $d_{j}$ also by $d_{k}^{*}$ if $\delta\left(d_{j}\right)=\delta\left(d_{k}\right)$ for $j \neq k$; thus, $\delta\left(d_{k}^{*}\right)=\delta\left(d_{k}\right)$ for all $k$. Define the function $v$ by

$$
v\left(d_{k}\right)=v(k)=\operatorname{sgn}\left|\begin{array}{ll}
a^{\prime}\left(d_{k}^{*}\right) & b^{\prime}\left(d_{k}^{*}\right) \\
a^{\prime}\left(d_{k}\right) & b^{\prime}\left(d_{k}\right)
\end{array}\right| .
$$

For a normal $\delta$ with $p=\delta(0)$ a positive outer point, the intersection sequence of $\delta$ with respect to $p$ is defined by the sequence $\left\{d_{k}\right\}$, the values of $d_{k}^{*}$ and the values of $v(k)$ for each $k$. A pair of normal representations $\delta$ and $\varepsilon$ have isomorphic intersection sequences if they have the same number of vertices, $d_{j}^{*}=d_{k}$ if $e_{j}^{*}=e_{k}$, and $v\left(d_{k}\right)=v\left(e_{k}\right)$. If two representations have isomorphic intersection sequences and one has an extension with certain topological properties, so will the other, as can be seen from the following theorem:

THEOREM [6, p. 49]. Let $\delta$ be a normal representation defined on a Jordan curve D; let $\varepsilon$ be normal and defined on the Jordan curve E. Suppose that each curve has a positive outer point and the curves have isomorphic intersection sequences. Then there exists a sense-preserving homeomorphism $h$ of $E^{2}$ onto $E^{2}$ taking $D$ onto $E$ such that $\varepsilon h=\delta$ (juxtaposition denotes function composition).

Thus, for our purposes two representations with isomorphic intersection sequences are interchangeable.

3. Interior boundaries. Let $D$ be an open subset of a two-dimensional manifold bounded by a finite number of Jordan curves and let $f$ be a mapping of $\bar{D}$ into $E^{2}$. If $f$ is light, open, and sense-preserving on $D$, and a local homeomorphism (relative to $\bar{D}$ ) at each point of Bdry $D$, then $f$ is called properly interior. We shall have use for a theorem of Stoïlow [3], [11, p. 88] which states that if $f$ is properly 
interior on $\bar{D}$, then at each point $p$ of $D$ there exists a closed two-cell neighborhood of $p$ on which $f$ is topologically equivalent to $w=z^{n}$ on the unit disk for some positive integer $n$.

A regular representation which has a properly interior extension to the disk is an interior boundary. The necessary and sufficient conditions given in [6] that a normal curve be an interior boundary are outlined in this section.

We have required that interior boundaries have sense-preserving light open extensions. It is well known that such representations have non-negative circulation; hence, the "cut" process described below only considers such representations. It will be necessary in $\$ 5$ to use the "cut" process for representations with nonpositive circulation; however, it will be apparent what adjustments should be made.

Suppose then that $\delta$ is a normal representation of a closed curve with nonnegative circulation. For such representations, $v\left(d_{0}\right)=1$. Since $v\left(d_{0}^{*}\right)=-v\left(d_{0}\right)$ $=-1$, there is some index $j$ such that $v(j)=-1$; let $k$ be the smallest such index. One then has the following cases: (I) $d_{k}^{*}<d_{k}$, (II) $d_{k}<d_{k}^{*}$; in the latter case one has for each $j<k$ the subcases: $\left(\mathrm{II}^{\prime}(\mathrm{j})\right) d_{j}<d_{k}<d_{k}^{*}<d_{j}^{*}$, (II"(j)) $d_{j}<d_{k}<d_{j}^{*}<d_{k}^{*}$.

For each $k$ and $j$ chosen as above ( $j$ is chosen only in case II) a "cut" will be defined. Each "cut" will lead to a pair of normal representations to be used as criteria for deciding if $\delta$ is an interior boundary.

Suppose $\delta$ and $\varepsilon$ are representations and $x=\delta(p)$ and $y=\delta(q)$ are two points such that $p<q$. Denote by $\delta(p) \delta(q)$ the representation obtained by restricting $\delta$ to $p \leqq t \leqq q$. If $x$ and $y$ each have only one pre-image, then $x y(\delta)$ or $x y$ will also be used to denote this representation. Let $-\delta$ be the representation gotten by tracing $\delta$ in the opposite direction. Consistent with this notation $\delta(q) \delta(p)(-\delta)$ traces from $\delta(q)$ to $\delta(p)$ via $-\delta$. We shall use $a p(\delta)+p b(\varepsilon)$ to denote the repre-

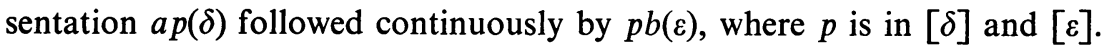

Given normal $\delta$ define the representations $\delta^{*}$ and $\delta^{* *}$ as follows (see Figures $2,3,4$ of [6, pp. 51-52]):

Case I

$$
\begin{aligned}
\delta^{*} & =\delta\left(d_{k}^{*}\right) \delta\left(d_{k}\right), \\
\delta^{* *} & =\delta(0) \delta\left(d_{k}^{*}\right)+\delta\left(d_{k}\right) \delta(2 \pi)
\end{aligned}
$$

Case II'(j).

$$
\begin{aligned}
\delta^{*} & =\delta\left(d_{j}\right) \delta\left(d_{k}\right)+\delta\left(d_{k}^{*}\right) \delta\left(d_{j}^{*}\right) \\
\delta^{* *} & =\delta(0) \delta\left(d_{k}^{*}\right)+\delta\left(d_{k}\right) \delta\left(d_{j}\right)(-\delta)+\delta\left(d_{j}^{*}\right) \delta(2 \pi)
\end{aligned}
$$

Case II"(j). $\delta^{*}$

$$
\begin{aligned}
& =\delta\left(d_{j}^{*}\right) \delta\left(d_{k}^{*}\right)+\delta\left(d_{k}\right) \delta\left(d_{j}\right)(-\delta) \\
\delta^{* *} & =\delta(0) \delta\left(d_{j}^{*}\right)+\delta\left(d_{j}\right) \delta\left(d_{k}\right)+\delta\left(d_{k}^{*}\right) \delta(2 \pi) .
\end{aligned}
$$


In all cases $\delta$ is an interior boundary if and only if $\delta^{*}$ and $\delta^{* *}$ are. Since $\delta^{*}$ and $\delta^{* *}$ are not normal, they are modified to normal representations $\bmod \delta^{*}$ and $\bmod \delta^{* *}[6$, p. 55 and p. 57]. The modifications are done so that $\delta$ is an interior boundary if and only if $\bmod \delta^{*}$ and $\bmod \delta^{* *}$ are interior boundaries, $\bmod \delta^{*}$ and $\bmod \delta^{* *}$ are normal and $\bmod \delta^{*}$ and $\bmod \delta^{* *}$ have strictly less vertices than $\delta$. In a finite number of steps the representation $\delta$ can be "cut" into Jordan curves so that $\delta$ is an interior boundary if and only if all the Jordan curves are interior boundaries, i.e., positively oriented.

4. An arc-lifting theorem. Theorem 1 will be a useful tool in the next section.

In this section a Jordan curve $J$ on an orientable two-dimensional manifold will be an oriented simple closed curve that bounds a two-cell. The two-cell bounded by $J$ will be denoted by Ins $J$. Jordan curves will be ordered by picking a fixed starting point and ordering the curve by positive traversal.

THEOREM 1. Let D be an open subset of an orientable two-dimensional manifold $M$ such that $\bar{D}$ is compact and let $J$ be a Jordan curve on $M$. Suppose $D$ is a subset of Ins $J(M-\operatorname{Ins} J)$ such that $J$ is a component of Bdry $D$. Let $f$ be a properly interior mapping of $\bar{D}$ into $E^{2}$ and let $f \mid J=\delta=u+i v$ be a regular representation. Suppose $\varepsilon(t)=a(t)+i b(t), 0 \leqq t \leqq 2 \pi$, is a regular representation of an arc in $E^{2}$ with $y=\varepsilon(q)$ for $0<q<2 \pi$. If either (1) $y=\delta(p)$ for some $p$ in $J$ and

$$
\operatorname{sgn}\left|\begin{array}{ll}
u^{\prime}(p) & v^{\prime}(p) \\
a^{\prime}(q) & b^{\prime}(q)
\end{array}\right|=-1(+1)
$$

or (2) there is a point $p$ in $D$ such that $f(p)=y$, then there exists an arc $B$ in $\bar{D}$ with end points $p$ and $b$ such that $B-\{p, b\} \subset D$ and such that $f$ maps $B$ homeomorphically into $[\varepsilon]$. In either case, if $b$ is in $D$, then $f(B)=[\varepsilon(0) \varepsilon(q)]$.

Proof. Suppose (1) holds. Select an open set $U$ in $D$ such that $f \mid \bar{U}$ is a homeomorphism, $\bar{U}-U$ is a Jordan curve, and $J \cap(\bar{U}-U)$ is an arc containing $p$ in its interior. If $J$ is given a positive orientation, then $\bar{U}-U$ is positively (negatively) oriented since $U$ is contained in $\operatorname{Ins} J(M-\operatorname{Ins} J)$. Because $f$ is a sense-preserving homeomorphism, $f(\bar{U}-U)$ is a positively (negatively) oriented Jordan curve. Choose $r$ and $s$ so that $0<r<q<s$ and $[\varepsilon(r) \varepsilon(s)] \cap f(\bar{U}-U)=\{y\}$. Let $x$ be any point with $r \leqq x<q$. From [5, Lemma 2, p. 1085] we have that

$$
w(f \mid \bar{U}-U, \varepsilon(s))-w(f \mid \bar{U}-U, \varepsilon(x))=\operatorname{sgn}\left|\begin{array}{l}
u^{\prime}(p) v^{\prime}(p) \\
a^{\prime}(q) \\
b^{\prime}(q)
\end{array}\right| .
$$

Because of $(1)$, the last term is $-1(+1)$. Since $f$ describes a positively (negatively) oriented Jordan curve, the only possible values of the index are $+1(-1)$ and 0 ; consequently, $w(f \mid \bar{U}-U, \varepsilon(x))=1(-1)$. This can only happen if $\varepsilon(x)$ is in $f(U)$; thus, $P=\{\varepsilon(t) \mid r \leqq t<q\}$ is contained in $f(U)$. Since $f$ is a homeomorphism 
on $\bar{U}$, there is an $\operatorname{arc} Q$ in $\bar{U}$ with end point $p$ mapping homeomorphically onto $P$ and $Q \cap(\bar{U}-U)=\{p\}$.

Let $P^{\prime}=[\varepsilon(0) \varepsilon(r)]$ and suppose $K$ is the component (in $D$ ) of $f^{-1}\left(P^{\prime}\right)$ containing $a$, the other end point of $Q$. Such a component is nondegenerate since $f$ is locally $z^{n}$ at $a$. The set $f(R)$ is an arc as it is a connected subset of [E]; thus $f(\bar{K})=[\varepsilon(z) \varepsilon(r)]$ for some $z$. Let $b$ be a pre-image of $\varepsilon(z)$ in $R$. Since $K \cup\{b\}$ is a nondegenerate connected set, there is an $\operatorname{arc} K^{\prime}$ in $K$ with end points $a$ and $b$. If $b$ is not in $\bar{D}-D$ and $f(b) \neq \varepsilon(0)$, there is an arc $A$ at $b$ mapping into $[\varepsilon(0) \varepsilon(z)]$ since $f$ is locally $z^{n}$ at $b$. Now $K$ is a component so $A$ must be contained in $K$; this is impossible since $f(K) \cap[\varepsilon(0) \varepsilon(z)]=\varnothing$. Therefore, if $b$ is not in $\bar{D}-D, f(b)=\varepsilon(0)$ and $f\left(Q \cup K^{\prime}\right)=[\varepsilon(0) \varepsilon(q)]$. Note that $f$ is homeomorphic on $Q \cup K^{\prime}\left[11\right.$, Theorem 4.1, p. 96]; hence $Q \cup K^{\prime}$ is the desired arc.

If (2) holds, take $K$ to be the component (in $D)$ of $f^{-1}([\varepsilon(0) \varepsilon(q)])$ containing $p$. An argument similar to that of the above paragraph produces the desired arc.

5. Interior mappings on the annulus. Let $A$ denote an open annulus in the plane bounded by Jordan curves $C_{1}$ and $C_{2}$, where $C_{1}$ is contained in Ins $C_{2}$. If $\delta$ and $\varepsilon$ are regular representations, we say $(\delta, \varepsilon)$ is an a-boundary when there exists a properly interior $f$ on $\bar{A}$ such that $f \mid C_{2}=\delta$ and $f \mid C_{1}=\varepsilon$.

LEMMA 5.1. Suppose $\delta$ is an interior boundary defined on a positively oriented Jordan curve $J$. Let $J=T_{1} \cup T_{2} \cup T_{3} \cup T_{4}$, where the $T_{i}$ are arcs which only intersect at the end points and the $T_{i}$ are numbered as $J$ is traversed in the positive order. If $\delta \mid T_{3}=-\left(\delta \mid T_{1}\right)$, then $\left(\delta \mid T_{2},-\left(\delta \mid T_{4}\right)\right)$ is an a-boundary.

Proof. Suppose without loss of generality that $J=\operatorname{Bdry}\{z|1 \leqq| z \mid \leqq 2$, $0 \leqq \arg z \leqq \pi\}$ and $D=$ Ins $J$. Let $f$ be a properly interior extension of $\delta$ on $\bar{D}$. It can also be assumed that $T_{1}=\{z$ in $J \mid z$ real, $1 \leqq z \leqq 2\}, T_{2}=\{z$ in $J|| z \mid=2\}$, $T_{3}=\{z$ in $J \mid z$ real, $-2 \leqq z \leqq-1\}$, and $T_{4}=\{z$ in $J|| z \mid=1\}$; also one can assume, in view of the hypothesis, that $f(x)=f(-x)$ for real $x$ in $J$.

Let $g(z)=z^{2}$ for $z$ in $\bar{D}$. Define $h$ to be $f g^{-1}$; note $h$ is well-defined and continuous on the annulus $\bar{A}=\{z|1 \leqq| z \mid \leqq 4\}$. Clearly $h$ is open at each point of $A$ except possibly at the real positive points of $A$; therefore, $h$ is open on $A$ $\left[8\right.$, Theorem 9, p. 336]. The mapping $h$ is the desired extension of $\left(\delta \mid T_{2},-\left(\delta \mid T_{4}\right)\right)$.

DEFINITION. Two normal representations $\delta$ and $\varepsilon$ intersect normally if $[\delta] \cap[\varepsilon]$ is a finite set, if no point of $[\delta] \cap[\varepsilon]$ is a vertex of either curve, and if the tangents to the curves at each point of intersection are linearly independent.

THEOREM 2. Let $\delta$ and $\varepsilon$ be normal representations of closed curves which intersect normally. Suppose $-\varepsilon$ is not an interior boundary. Then $(\delta, \varepsilon)$ is an a-boundary if and only if one of the following holds:

(1) Suppose $\varepsilon$ has some points of positive circulation. Let $p$ be a point not in $[\varepsilon]$ such that $w(\varepsilon, p)>0$. Suppose $\phi$ represents an arc which intersects $\delta$ and $\varepsilon$ normally and which has one end point at $p$ and the other at a point $q$ in the 
unbounded component of $E^{2}-[\delta]-[\varepsilon]$. If $[\phi] \cap[\delta]=\left\{a_{1}, \cdots, a_{m}\right\}$ and $[\phi] \cap[\varepsilon]=\left\{b_{1}, \cdots, b_{n}\right\}$, then the curve

$$
\zeta^{i j}=\delta(0) a_{i}+a_{i} b_{j}(\phi)+b_{j} b_{j}(-\varepsilon)+b_{j} a_{i}(-\phi)+a_{i} \delta(0)
$$

is an interior boundary for some $i$ and $j, 1 \leqq i \leqq m, 1 \leqq j \leqq n$.

(2) Suppose $\varepsilon$ has nonpositive circulation and $\varepsilon$ has a cut of Type I at $\varepsilon_{r}=\varepsilon\left(e_{k}\right)$ with $e_{k}^{*}<e_{k}$. Then either

(a) there is a point $p$ in $[\delta] \cap\left[\varepsilon\left(e_{k}^{*}\right) \varepsilon\left(e_{k}\right)\right]$ such that $\zeta^{n}$ is an interior boundary for some $n, 0 \leqq n \leqq w^{+}(\delta, p)+w^{+}(-\varepsilon, p)$, where $\zeta^{0}=\delta(0) p+p \varepsilon\left(e_{k}\right)$ $+\varepsilon\left(e_{k}\right) \varepsilon\left(e_{k}\right)(-\varepsilon)+\varepsilon\left(e_{k}\right) p(-\varepsilon)+p \delta(0)$ and $\zeta^{n}=\delta(0) p+p \varepsilon\left(\varepsilon_{k}\right)+\sum_{i=1}^{n} \varepsilon\left(e_{k}^{*}\right) \varepsilon\left(e_{k}\right)$ $+\varepsilon\left(e_{k}\right) \varepsilon\left(e_{k}\right)(-\varepsilon)+\sum_{i=1}^{n} \varepsilon\left(e_{k}\right) \varepsilon\left(e_{k}^{*}\right)(-\varepsilon)+\varepsilon\left(e_{k}\right) p(-\varepsilon)+p \delta(0)$.

(b) $\varepsilon^{*}$ represents a negatively oriented Jordan curve and $\left(\delta, \varepsilon^{* *}\right)$ is an aboundary.

(3) Suppose $\varepsilon$ has non positive circulation and $\varepsilon$ has a cut of Type II at $\varepsilon_{r}=\varepsilon\left(e_{k}\right)$ with $e_{k}<e_{k}^{*}$. Then either

(a) there exists a point $p$ in $[\delta] \cap\left[\varepsilon(0) \varepsilon\left(e_{k}\right)\right]$ such that $\zeta=\delta(0) p+p \varepsilon\left(e_{k}\right)$ $+\varepsilon_{r} \varepsilon_{r}(-\varepsilon)+\varepsilon\left(e_{k}\right) p(-\varepsilon)+p \delta(0)$ is an interior boundary;

(b) if $\phi$ represents an arc with end point $\varepsilon(0)$, in the unbounded component of $E^{2}-[\varepsilon]$, and such that $\phi$ and $\delta$ intersect normally at $\left\{a_{1}, \cdots, a_{m}\right\}$, then the curve $\zeta^{i}=\delta(0) a_{i}+a_{i} \varepsilon(0)(\phi)+\varepsilon(0) \varepsilon\left(e_{k}\right)+\varepsilon_{r} \varepsilon_{r}(-\varepsilon)+\varepsilon\left(e_{k}\right) \varepsilon(0)(-\varepsilon)+\varepsilon(0) a_{i}(-\phi)$ $+a_{i} \delta(0)$ is an interior boundary for some $i, 1 \leqq i \leqq m$;

(c) $-\varepsilon^{*}$ is an interior boundary and $\left(\delta, \varepsilon^{* *}\right)$ is an a-boundary;

(d) $-\varepsilon^{* *}$ is an interior boundary and $\left(\delta, \varepsilon^{*}\right)$ is an a-boundary.

Necessity proof. Let $f$ be a properly interior extension of $(\delta, \varepsilon)$ on $\bar{A}$.

Suppose $\varepsilon$ has some points of positive circulation and let $p, q$, the $a_{i}$, and the $b_{i}$ be as in (1) of the theorem. Let $\varepsilon=u(t)+i v(t)$ and $\phi=a(t)+i b(t)$ be parametrized on $C_{1}$ so that the pre-image of $b_{i}$ under $\varepsilon$ and $\phi$ is $t_{i}, 1 \leqq i \leqq n$. Define $\sigma_{i}$ by

$$
\sigma_{\iota}=\operatorname{sgn}\left|\begin{array}{ll}
u^{\prime}\left(t_{i}\right) & v^{\prime}\left(t_{i}\right) \\
a^{\prime}\left(t_{i}\right) & b^{\prime}\left(t_{i}\right)
\end{array}\right| .
$$

Let $\phi$ be ordered as it increases from $p$ to $q$; assume $b_{i} \leqq b_{j}$ if and only if $i \leqq j$. Since $q$ is in the unbounded component of $E^{2}-[\delta]-[\varepsilon], w(\varepsilon, q)=0$; thus $\sum_{i=1}^{n} \sigma_{i}=w(\varepsilon, p)[5$, Lemma 2, p. 1085]. By hypothesis, this last quantity is strictly positive. For each $j$ such that $\sigma_{j}=1$, Theorem 1 can be applied at $t_{j}$, yielding an $\operatorname{arc} B_{j}$ with end points $t_{j}$ and $x_{j}$ and which maps into $\left[b_{j} q(\phi)\right]$. If $x_{j}$ were in $A$, then $x_{j}$ would map onto $q$. This is not possible, since no point of $A$ can map into the unbounded component of $E^{2}-[\delta]-[\varepsilon]$ under a properly interior mapping; thus, $x_{j}$ is in $C_{1}$ or $C_{2}$. If $x_{j}$ is in $C_{1}$, then $x_{j}=t_{k}$ for some $k$ and $\sigma_{k}=-1$. If every $x_{j}$ is in $C_{1}$, then there are at least as many $\sigma_{k}=-1$ as $\sigma_{j}=1$. This contradicts the fact that $\sum_{i=1}^{n} \sigma_{i}>0$; hence, some $x_{j}$ is in $C_{2}$ and 
$f\left(x_{j}\right)=a_{i}$ for some $i, 1 \leqq i \leqq m$. Suppose $D$ and $g$ are defined as in the proof of Lemma 5.1 and $W=\{z|1<| z \mid<4\}$. By composing $f$ with an appropriate homeomorphism of $\bar{W}$ onto the domain of $f$, we may assume that the domain of $f$ is $\bar{W}$ and $B_{j}=\{z|1<| z \mid<4, \arg z=0\}$. The mapping $f g$ is properly interior on $\bar{D}$ and extends the curve $\zeta^{i j}$ of (1).

Suppose $\varepsilon$ has nonpositive circulation and $\varepsilon$ has a cut of Type I at some $\varepsilon_{r}=\varepsilon\left(e_{k}\right)$. By definition of a Type I cut, $v\left(e_{k}\right)=+1$ with $e_{k}^{*}<e_{k}$. Recall that $\varepsilon^{*}=\varepsilon\left(e_{k}^{*}\right) \varepsilon\left(e_{k}\right)$; $\varepsilon^{*}$ represents a negatively oriented Jordan curve $[6$, Lemma 5, p. 53]. Choose $p$ on $\left[\varepsilon^{*}\right]$ with $p \neq e_{r}$. Since $v\left(e_{k}\right)=+1$, by Theorem 1 there is an arc $L_{1}^{\prime}$ with end point at $e_{k}^{*}$ which maps into $\left[p \varepsilon\left(e_{k}\right)(\varepsilon)\right]$. If the other end point $z$ of $L_{1}^{\prime}$ is not in $\bar{A}-A$, then $f(z)=\varnothing$. Again by Theorem 1 , there must be an $\operatorname{arc} L_{1}^{\prime \prime}$ with end point at $z$ which maps into $\left[\varepsilon\left(e_{k}^{*}\right) p(\varepsilon)\right]$. Let $L_{1}=L_{1}^{\prime} \cup L_{1}^{\prime \prime}$. If the other end point $x$ of $L_{1}$ is in $A$, we apply this process again, obtaining an $\operatorname{arc} L_{2}$ with one end point at $x$ and which maps into $\left[\varepsilon^{*}\right]$. The process must terminate after a finite number of steps since properly interior mappings are finite-to-one. Thus there exists an $\operatorname{arc} K=L_{1} \cup L_{2} \cup \cdots \cup L_{s}$ where each $L_{i}$ maps onto the Jordan curve determined by $\varepsilon^{*}$ for $1 \leqq i<s$. One end point of $K$ is $e_{k}^{*}$; the other, $y$, is either in $C_{1}$ or $C_{2}$.

If $y$ is in $C_{2}$, define $W, D$, and $g$ as before. Assume the domain of $f$ is $\bar{W}$ and $K=\{z|1<| z \mid<4, \arg z=0\}$. Then $f g$ is a properly interior extension of $\zeta^{s-1}$ in (2a). For each $L_{i}$, there must be points near $L_{i}$ mapping onto points near $p=f(y)$. The number of pre-images of a point $p^{\prime}$ not in $[\delta]$ or $[\varepsilon]$ is $w\left(\delta, p^{\prime}\right)+w\left(-\varepsilon, p^{\prime}\right)\left[2\right.$, p. 72]. If $p^{\prime}$ is near $p$, then $w\left(\delta, p^{\prime}\right) \leqq w^{+}(\delta, p)$ and $w\left(-\varepsilon, p^{\prime}\right) \leqq w^{+}(-\varepsilon, p)$. Thus $s-1 \leqq w^{+}(\delta, p)+w^{+}(-\varepsilon, p)$.

If $y$ is in $C_{1}, y=e_{k}$ since $f$ is a local homeomorphism on $C_{1}$. Let $J$ be the positively oriented Jordan curve determined by $K$ and $V=\left\{t\right.$ in $\left.C_{1} \mid e_{k}^{*} \leqq t \leqq e_{k}\right\}$ and let $J^{\prime}$ be the positively oriented Jordan curve determined by $K$ and $C_{1}-V$. Either Ins $J$ is contained in $A$ or Ins $J$ contains the points of $C_{1}-V$. Suppose by way of contradiction that the latter case occurred. Let $T$ be the unit circle, let $U_{1}=\{z$ in $\operatorname{Ins} T \mid \operatorname{Im} z>0\}$, and let $U_{2}=\{z$ in $\operatorname{Ins} T \mid \operatorname{Im} z<0\}$. There exists a homeomorphism $h$ on $\bar{U}_{2} \cup T$ such that $h(T)=-C_{1}, h\left(\operatorname{Bdry} U_{1}\right)=-J$, $h\left(U_{2}\right)=\operatorname{Ins} J^{\prime}$, and $h$ is sense-preserving on $U_{2}$. The mapping $f h$ is light open on $U_{2}$. Since $f$ maps $J$ onto a negatively oriented Jordan curve, $f$ h maps Bdry $U_{1}$ onto a positively oriented Jordan curve; thus, on Bdry $U_{1}, f h$ is topologically equivalent to $w=z^{s+1}[11$, Theorem 4.3, p. 86]. Define $f h$ to be topologically equivalent to $w=z^{s+1}$ on $\bar{U}_{1}$; then $f h$ is light open on Ins $T$ [8, Theorem 9, p. 336]. Since $f h|T=f|-C_{1}=-\varepsilon$, the curve $-\varepsilon$ is an interior boundary. This is contrary to hypothesis; thus Ins $J$ is contained in $A$.

On $J$ the mapping $f$ is topologically equivalent to $w=z^{s+1}$ on the unit circle $[11$, Theorem 4.3, p. 86]; hence, $f$ can be defined on $J \cup$ Ins $J$ to be topologically equivalent to $w=z^{s+1}$ on the unit disk. Thus there are arcs $X$ and $Y$ in Ins $J$ such that $X$ has end point $e_{k}^{*}, Y$ has end point $e_{k}, X$ and $Y$ intersect only at the other end point, $f$ is a homeomorphism on $X$ and on $Y$, and $f(X)=f(Y)$. Let $A_{1}$ 
be the open annulus bounded by $C_{2}$ and by the Jordan curve determined by $X, Y$, and $C_{1}-V$. There exists a map $h$ from $\bar{A}_{1}$ onto $\{z|1 \leqq| z \mid \leqq 2\}$ such that $h$ is a homeomorphism on $\bar{A}_{1}-X-Y$, on $X$, and on $Y$. Also $h(X)=h(Y)$ $=\{z|1 \leqq| z \mid \leqq 3 / 2, \arg z=0\}$ and, for $x$ in $X$ and $y$ in $Y, h(x)=h(y)$ if and only if $f(x)=f(y)$. Clearly $f h^{-1}$ is well-defined, continuous everywhere, light, and open except possibly at $h(X)$; therefore, $f h^{-1}$ is light open on $h(X)$ [8, Theorem 9 , p. 336]. The mapping $f h^{-1}$ is a properly interior extension of $\left(\delta, \varepsilon^{* *}\right)$; thus $\left(\delta, \varepsilon^{* *}\right)$ is an a-boundary. Since $\varepsilon^{*}$ describes a negatively oriented Jordan curve, $-\varepsilon^{*}$ is an interior boundary. This gives case $(2 b)$.

Suppose $\varepsilon$ has nonpositive circulation and $\varepsilon$ has a cut of Type II at some $\varepsilon_{r}=\varepsilon\left(e_{k}\right)$. Select $k$ to be the smallest integer such that $v\left(e_{k}\right)=1$. Let $\phi$ be a representation as described in (3b) of the theorem; let $V=[\phi] \cup\left[\varepsilon(0) \varepsilon\left(e_{k}\right)(\varepsilon)\right]$. Since $v\left(e_{k}\right)=1$, it follows from Theorem 1 that there is an $\operatorname{arc} K$ with end point $e_{k}^{*}$ mapping into $V$. If the other end point $x$ of $K$ is in $A, K$ maps onto $V$; however, this is not possible since a properly interior mapping cannot map points of $A$ into the unbounded component of $E^{2}-[\delta]-[\varepsilon]$. Hence, $x$ is in $C_{1}$ or $C_{2}$.

If $x$ is in $C_{2}$, define $W, D$, and $g$ as before. Once again we may assume the domain of $f$ is $\bar{W}$ and $K=\{z|1<| z \mid<4, \arg z=0\}$. If $f(x)=p$ is a point of $\left[\varepsilon(0) \varepsilon\left(e_{k}\right)\right]$, then $f g$ is a properly interior extension on $\bar{D}$ of $\zeta$ in (3a). If $f(x)=a_{i}$ is a point of $[\phi]$, then $f g$ is a properly interior extension on $\bar{D}$ of $\zeta^{i}$ in (3b).

Suppose $x$ is in $C_{1}$. Since $f$ is a homeomorphism at $x, f(x)$ must be a vertex $\varepsilon_{p}$. By definition of $V, p \leqq r$; however, for Type II cuts it must be that $p<r$. Let $\varepsilon_{p}=\varepsilon\left(e_{j}\right)$ with $e_{j}<e_{k}<e_{k}^{*}$. Note that $x=e_{j}^{*}$. Assume that $e_{k}^{*}<e_{j}^{*}$; the proof for $e_{j}^{*}<e_{k}^{*}$ is similar. Let $J$ be the Jordan curve determined by $K$ and $\left\{t \mid e_{k}^{*} \leqq t \leqq e_{j}^{*}\right\}$ and let $L$ be the Jordan curve determined by $K$ and $C_{1}-J$. Orient these curves by the orientation of $C_{1}$. By definition of Type II cuts, $f \mid J=\varepsilon^{*}$ and $f \mid L=\varepsilon^{* *}$. Either Ins $J$ or Ins $L$ is a disk contained in $A$. If Ins $J$ is a disk contained in $L$, the restriction of $f$ to Ins $J$ gives a light open extension of $\varepsilon^{*}$. But $J$ is negatively oriented; hence, $-\varepsilon^{*}$ is an interior boundary. The restriction of $f$ to the annulus bounded by $C_{2}$ and the positively oriented Jordan curve $L$ extends $\left(\delta, \varepsilon^{* *}\right)$; thus, $\left(\delta, \varepsilon^{* *}\right)$ is an $a$-boundary. This is case (3c). If Ins $L$ is a disc contained in $A$, a similar argument shows that $-\varepsilon^{* *}$ is an interior boundary and $\left(\delta, \varepsilon^{*}\right)$ is an $a$-boundary. This gives case (3d), completing the necessity proof.

Sufficiency proof. If condition (1), (2a), (3a), or (3b) holds, it follows from Lemma 5.1 that $(\delta, \varepsilon)$ is an $a$-boundary.

Suppose that $(2 \mathrm{~b})$ holds where $\varepsilon$ has a cut of Type I. Then $\left(\delta, \varepsilon^{* *}\right)$ is an $a$ boundary; also, $\varepsilon^{*}$ describes a negatively oriented Jordan curve. Number the vertices of $\varepsilon^{* *}$ as if they were vertices of $\varepsilon$. The point $\varepsilon_{r}$ is not a vertex of $\varepsilon^{* *}$. Recall that $\left[\varepsilon^{*}\right]$ intersects $\left[\varepsilon^{* *}\right]$ only in the point $\varepsilon_{r}[6$, Lemma 5, p. 53$]$.

Let $f$ be a properly interior extension of $\left(\delta, \varepsilon^{* *}\right)$ on $A$. Choose an arc $B$ in Ins [ $\left.\varepsilon^{*}\right]$ with end point at $\varepsilon_{r}$. By Theorem 1 there is an $\operatorname{arc} K$ in $A$ with end point 
on $C_{1}$ mapping homeomorphically onto $B$. Let $A_{1}=\{z|1<| z \mid<2\}$, $X=\{z|| z \mid=1,-\pi / 2 \leqq \arg z \leqq 0\}$, and $Y=\{z|| z \mid=1,0 \leqq \arg z \leqq \pi / 2\}$. There exists a mapping $h_{1}$ from $\bar{A}_{1}$ to $\bar{A}$ such that $h$ is a homeomorphism on $\bar{A}_{1}-X-Y$, on $X$, and on $Y$; also, $h_{1}(X)=h_{1}(Y)=K$. Let $L=\{z|0 \leqq| z \mid \leqq 1$, $z$ imaginary $\}$ and let $U$ be the domain bounded by $X, Y$, and $L$. There exists a mapping $h_{2}$ properly interior on $\bar{U}$, except at $z=1$, which maps $L$ onto [ $\varepsilon^{*}$ ] and such that $h_{2}\left|X=f h_{1}\right| X$ and $h_{2}\left|Y=f h_{2}\right| Y$. Define $h$ on $\bar{A}_{1} \cup \bar{U}$ by $h \mid \bar{U}=h_{2}$, $h \mid \bar{A}_{1}=f h_{1}$. Then $h$ is a properly interior extension of $(\delta, \varepsilon)$.

Suppose that (3c) holds and $\varepsilon$ has a cut of Type II' (j) or Type II" (j) at $\varepsilon_{r}$. The $\operatorname{arc} T=\left[\varepsilon\left(e_{j}\right) \varepsilon_{r}(\varepsilon)\right]$ is traced in opposite directions by $\varepsilon^{*}$ and $\varepsilon^{* *}$; hence, in the same direction by $-\varepsilon^{*}$ and $\varepsilon^{* *}$.

Let $A_{1}, X, Y$, and $U$ be defined as before. Choose $f$ a properly interior extension of $\left(\delta, \varepsilon^{* *}\right)$ on $\bar{A}_{1}$. Let $V=X \cup Y$ and suppose without loss of generality that $V$ is the arc mapped onto $T$ by $f$. Since $-\varepsilon^{*}$ is an interior boundary which traces $T$ in the same direction as $f \mid V$, there exists a properly interior extension $g$ of $-\varepsilon^{*}$ on $U$ such that $V$ is mapped onto $T$. Define $h$ on $\bar{A}_{1} \cup \bar{U}$ by $h \mid \bar{A}_{1}=f$ and $h \mid \bar{U}=g$. Then $h$ is properly interior on $\bar{A}_{1} \cup \bar{U}$ and extends $(\delta, \varepsilon)$.

The proof for ( $3 \mathrm{~d})$ is similar.

This completes the proof of the theorem.

Definition. Let $\delta$ be a representation of a closed curve. An arc $B$ is an interior arc of $\delta$ with end point $p$ if $p$ is one end point of $B,[\delta] \cap B=\{p\}$, and $w\left(\delta, p^{\prime}\right)=w^{+}(\delta, p)$ for all $p^{\prime}$ in $B$.

LemMA 5.2. Suppose $\delta$ and $\varepsilon$ are normal interior boundaries with $p$ a point of $[\delta]$ and $q$ a point of $[\varepsilon]$, where neither $p$ nor $q$ is a vertex. If $\phi$ represents an arc $B$ from $p$ to $r$ such that $B$ is an interior arc of $\delta$ at $p$ and $[q r(\phi)]$ is an interior arc of $\varepsilon$ at $q$, then $(\delta,-\varepsilon)$ is an a-boundary.

Proof. Let $D$ be a disk divided into disks $G$ and $H$ by an arc $E$. From [6, Lemma 6 , p. 53] we see that $\delta^{\prime}=\delta(0) p+\phi+(-\phi)+p \delta(0)$ has extension $g$ on $\bar{G}$ and $\varepsilon^{\prime}=\varepsilon(0) q+q r(\phi)+\dot{r} q(-\phi)+q \varepsilon(0)$ has extension $h$ on $\bar{H}$ such that $g$ and $h$ are light open and properly interior except at the points of Bdry $G$ and Bdry $H$ mapping onto $r$. Without loss of generality assume that $h|E=g| E=q r(\phi)$ $+r q(-\phi)$. There must be arcs $U$ and $V$ on Bdry $G$ such that $g \mid U=p q(\phi)$ and $g \mid V=q p(-\phi)$. Define $f$ on $\bar{D}$ by $f \mid \bar{G}=g$ and $f \mid \bar{H}=h$; then $f$ is properly interior on $\bar{D}[8$, Theorem 9 , p. 336]. The conclusion follows from an application of Lemma 5.1 to $f \mid$ Bdry $D$.

LEMMA 5.3. If $(\delta, \varepsilon)$ is an a-boundary, then so is $(-\varepsilon,-\delta)$.

Proof. Choose $f$ a properly interior extension of $(\delta, \varepsilon)$ on $A$. There exists a homeomorphism $h$ on $A$ topologically equivalent to $1 / z$ on $\{z \mid 1 \leqq z \leqq 2\}$ such that $h \mid C_{1}=-C_{2}$ and $h \mid C_{2}=-C_{1}$. The mapping $f h$ extends $(-\varepsilon,-\delta)$. 
THEOREM 3. Let $\delta$ and $\varepsilon$ be normal interior boundaries which intersect normally.

(1) Suppose $[\delta] \cap[\varepsilon]=\varnothing$. Then $(\delta,-\varepsilon)$ is an a-boundary if and only if $[\varepsilon]$ is contained in a component $P$ of $E^{2}-[\delta]$ such that $w(\delta, p)>0$ for $p$ in $P$ or $[\delta]$ is contained in a component $Q$ of $E^{2}-[\varepsilon]$ such that $w(\varepsilon, q)>0$ for $q$ in $Q$.

(2) If $[\delta] \cap[\varepsilon] \neq \varnothing$, then $(\delta,-\varepsilon)$ is an a-boundary.

Proof. Case 1. Suppose $(\delta,-\varepsilon)$ is an $a$-boundary. Either $[\delta]$ is contained in $U$, the unbounded component of $E^{2}-[\varepsilon]$, or $[\varepsilon]$ is contained in the unbounded component of $E^{2}-[\delta]$ (or both). Suppose the former holds. Let $J$ be a Jordan curve in $U$ such that $[\varepsilon]$ is in Ins $J$ and $[\delta]$ is in the other component of $E^{2}-J$. Now there must be a point $y$ of $f(A)$ on $J$; otherwise, $f(A)$ would not be connected. Thus the number of pre-images of $y$ in $A, n(y)$, is strictly positive. By [2, p. 72], $n(y)=w(\delta, y)+w(-\varepsilon, y)$ and since $y$ is in $U, w(-\varepsilon, y)=0$. Index is constant on components; thus, $w(\delta, p)=w(\delta, y)=w(\delta, y)+w(-\varepsilon, y)=n(y)>0$ for any $p$ in the component $P$ of $E^{2}-[\delta]$ containing $y$. Since $[\varepsilon]$ is contained in $P$ the conclusion follows. The proof for the case where $[\varepsilon]$ is contained in the unbounded component of $E^{2}-[\delta]$ is similar.

Now suppose $[\varepsilon]$ is in a component $P$ of $E^{2}-[\delta]$ such that $w(\delta, p)>0$ for $p$ in $P$. Let $\phi$ represent an arc with one end point at $r$ in $P$, the other at $s$ in the unbounded component of $E^{2}-[\delta]$, such that $\phi$ and $\delta$ intersect normally, $[\phi] \cap[\varepsilon]=\{q\}$, and $q r(-\phi)$ is an interior arc of $\varepsilon$ at $q$. Let $h$ be a properly interior extension of $\delta$ on the unit disk $D$. Since $w(\delta, r)>0, r$ has a pre-image $x$ in $D[2$, p. 72]. By Theorem 1 , there is an arc $K$ with end point $x$ mapping into [ $\phi]$. The other end point $y$ of $K$ must be in Bdry $D$ since $K$ cannot map onto $[\phi]$. Let $p=h(y)$ and $\zeta=p r(-\phi)$. Without loss of generality we may assume that $K=\{z|0<| z \mid<1, \arg z=0\}$. Let $W=\{z|| z \mid<1, \operatorname{Im} z>0\}$ and $g(z)=z^{2}$ for $z$ in $W$. Then $h g$ is a light open extension of $\delta(0) p+\zeta+(-\zeta)$ $+p \delta(0)$. The proof now proceeds exactly as the proof of Lemma 5.2, yielding the result that $(\delta,-\varepsilon)$ is an $a$-boundary.

If $[\delta]$ is in a component of $E^{2}-[\varepsilon]$ on which the index of $\varepsilon$ is strictly positive, the above argument shows that $(\varepsilon,-\delta)$ is an $a$-boundary. By Lemma $5.3,(\delta,-\varepsilon)$ is then an $a$-boundary. This completes the proof for Case 1.
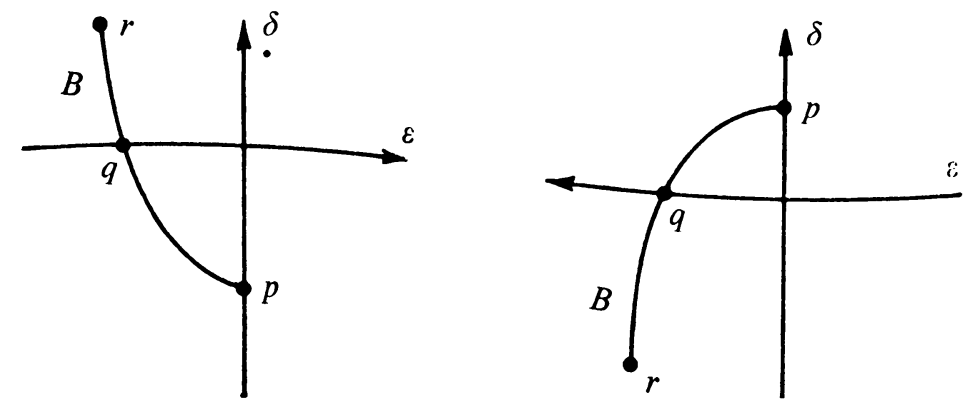

FIGURE 1 
Case 2. As Figure 1 shows, there always exists an arc $B$ with a representation $\phi$ satisfying the hypothesis of Lemma 5.2 when $[\delta] \cap[\varepsilon] \neq \varnothing$. That $B$ and $[q r(\phi)]$ are interior arcs is seen immediately from [5, Lemma 2, p. 1085]. The conclusion then follows from Lemma 5.2.

REMARK. Let $\delta$ and $\varepsilon$ be a pair of normal representations which intersect normally. It is desired to determine if $(\delta, \varepsilon)$ is an $a$-boundry. If both $\delta$ and $-\varepsilon$ are interior boundaries, apply Theorem 3 . If $\delta$ is not an interior boundary, and $-\varepsilon$ is, test $(-\varepsilon,-\delta)$; by Lemma 5.3 , this is the same as testing $(\delta, \varepsilon)$. So we may assume that $-\varepsilon$ is not an interior boundary. Then Theorem 2 can be applied to $(\delta, \varepsilon)$. If condition (1), (2a), (3a), or (3b) holds, the curve that arises can be modified to be normal and tested by the methods of [6]. If condition (2b), (3c) or (3d) holds, then $\varepsilon^{*}$ and $\varepsilon^{* *}$ are modified $\left[6\right.$, p. 55 and p. 57] to normal curves $\bmod \varepsilon^{*}$ and $\bmod \varepsilon^{* *}$ with strictly less vertices than $\varepsilon$. For any curve $\zeta,(\delta, \zeta)$ is an $a$-boundary if and only if $(\delta, \bmod \zeta)$ is; $\zeta$ is an interior boundary if and only if $\bmod \zeta$ is. Thus Theorem 2 is repeatedly applied until either case (1), (2a), (3a), or (3b) arises or until $\varepsilon$ is "cut" into a Jordan curve $J$. It is then desired to test $(\delta, J)$. If $J$ is positively oriented, (1) of Theorem 2 applies. If $J$ is negatively oriented, test $(-J,-\delta)$.

\section{BIBLIOGRA PHY}

1. M. Heins and M. Morse, Deformation classes of meromorphic functions and their extensions to interior transformations, Acta Math. 80 (1947), 51-103.

2. M. Morse, Topologicalmethods in the theory of functions of a complex variable, Annals of Mathematics Studies No. 15, Princeton Univ. Press, Princeton, N. J., 1947.

3. S. Stoïlow, Lecons sur les principes topologiques de la théorie des fonctions analytiques, Gauthier-Villars, Paris, 1938.

4. C. J. Titus, The image of the boundary under a local homeomorphism, Lectures on Functions of a Complex Variable, Univ. of Michigan Press, Ann Arbor, Mich., 1955.

5. - A theory of normal curves and some applications, Pacific J. Math. 10 (1960), 1083-1096.

6. - The combinatorial topology of analytic functions on the boundary of a disk, Acta Math. 106 (1961), 45-64.

7. C. J. Titus and G. S. Young, An extension theorem for a class of differential operators, Michigan Math. J. 6 (1959), 195-204.

8. - The extension of interiority, with some applications, Trans. Amer. Math. Soc. 103 (1962), 329-340.

9. H. Whitney, Differentiable manifolds, Ann. of Math. 37 (1936), 645-680.

10. - On regular closed curves in the plane, Compositio Math. 4 (1937), 276-284.

11. G. T. Whyburn, Topological analysis, Princeton Univ. Press, Princeton, N. J., 1958.

TULANE UNIVERSITY,

New ORLeans, Louisiana 